\title{
Herbicidas no alto Rio Poxim, Sergipe e os riscos de contaminação dos recursos hídricos ${ }^{1}$
}

\author{
Herbicides in the upper Poxim River, Sergipe, and the risk of contamination of water \\ resources
}

\author{
Fábio Brandão Britto ${ }^{2 *}$, Anderson Nascimento do $\operatorname{Vasco}^{3}$, Ana Paula Sousa Pereira ${ }^{2}$, Arisvaldo Vieira Méllo \\ Júnior $^{4}$ e Luis Carlos Nogueira ${ }^{5}$
}

\begin{abstract}
Resumo - A crescente atividade agrícola em áreas de mananciais tem causado preocupações quanto à contaminação por herbicidas em áreas agrícolas. O problema se torna mais importante quando a contaminação pode afetar a água para uso humano, como ocorre com a água do Rio Poxim, que abastece a cidade de Aracaju, capital do Estado de Sergipe. O objetivo do estudo foi avaliar o risco de contaminação de águas superficiais e subterrâneas por herbicidas no alto da Sub-bacia do Rio Poxim e detectar a presença dos princípios ativos Diuron e Ametrina, à montante das plantações de cana-de-açúcar. A análise de risco foi realizada mediante critérios da Environmental Protection Agency (EPA), índice de GUS e método de GOSS. Observou-se que vários princípios ativos sofrem risco de lixiviação, demonstrando a importância do monitoramento do rio para controle tanto da qualidade da água como da frequência e volume de herbicidas aplicado na região. A partir do resultado, foi realizado um monitoramento bimensal de julho de 2009 a julho de 2010, em dois pontos de amostragem. As amostras de água foram analisadas em laboratório, onde se, constatou a presença de Diuron e Ametrina. A qualidade da água na Sub-bacia do Rio Poxim está sendo influenciada pelo uso de herbicidas na região. Ocorreu um aumento nas concentrações dos herbicidas na água superficial, durante o período chuvoso, provocado possivelmente pelo escoamento superficial.
\end{abstract}

Palavras-chave - Contaminação de águas superficiais e subterrâneas. Efeito dos herbicidas. Solos-lixiviação.

\begin{abstract}
Increased agricultural activity in watershed areas has been causing concern over contamination by herbicides in agricultural areas. The problem becomes more important when contamination can affect water for human consumption, as happens with water from the Poxim river, which supplies the city of Aracaju, capital of the State of Sergipe. The aim of this study was to evaluate the risk of contamination by herbicides to both surface and groundwater in the upper sub-basin of the Poxim River, and to detect the presence of the active ingredients Diuron and Ametrine up-river from the sugar-cane plantations. Risk analysis was carried out using criteria from the Environmental Protection Agency (EPA), the GUS index, and the GOSS method. It was observed that several active ingredients are at risk of leaching, demonstrating the importance of monitoring the river to control both the quality of water and the frequency and volume of herbicides used in the region. Based on the results, monitoring was carried out bi-monthly from July 2009 to July 2010 at two sampling points. Water samples were analyzed in the laboratory, where the presence of Diuron and Ametrine was noted. Water quality in the Sub-basin of the Rio Poxim is being influenced by the use of herbicides in the region. There was an increase in herbicide concentration in the surface water during the rainy season, possibly caused by soil runoff.
\end{abstract}

Key words - Contamination of surface and groundwater. Effect of herbicides. Soil-leaching.

\footnotetext{
*Autor para correspondência

${ }^{1}$ Recebido para publicação em 14/03/2011; aprovado em 03/10/2011

Parte da Dissertação de Mestrado do primeiro autor, apresentada ao Programa de Pós-Graduação em Agroecosistemas/NEREN, Universidade Federal de Sergipe/UFS

${ }^{2}$ Programa de Pós-Graduação em Agroecossitema/NEREN/Universidade Federal de Sergipe/UFS, São Cristovão-SE, Brasil, 49.100-000, brandaobritto@hotmail.com, bioanap_va@hotmail.com

${ }^{3}$ Universidade Federal de Sergipe, São Cristovão-SE, Brasil, 49.100-000, anderovasco@ yahoo.com.br

${ }^{4}$ Departamento de Hidráulica/USP, Av. Prof. Almeida Prado 83, Travessa 2, São Paulo-SP, 05.508-900, arimellojr@ gmail.com

${ }_{5}^{5}$ Pesquisador da Embrapa Cocais e Planícies Inundáveis, São Luís-MA, Brasil, 65.046-660, lcnogueira@gmail.com
} 


\section{Introdução}

O aumento da atividade agrícola desenvolvida em áreas de mananciais tem causado preocupação quanto ao potencial de contaminação decorrente da aplicação de herbicidas. Sabe-se que a agricultura é uma das possíveis fontes de contaminação ambiental, geralmente apontada como importante contribuinte de poluentes (BRITO et al., 2001; GRÜTZMACHER et al., 2008).

Um dos principais problemas decorrente do crescimento das fontes de poluição pontuais e difusas é o risco de deterioração dos recursos hídricos. Isso pode ocorrer no cultivo da cana-de-açúcar, pois o uso constante de herbicidas pré e pós-emergentes potencializa o risco de contaminação das águas, e exige estudos locais do comportamento desses produtos no solo (MATALLO, 2003). O problema se agrava quando as áreas de recarga, constituídas por materiais arenosos, estão sendo ocupadas por atividades agrícolas com número expressivo de aplicações de pesticidas (GOMES et al., 2001).

Quando aplicados diretamente no solo, os herbicidas podem ser degradados por vias químicas ou pela ação de microorganismos. Entretanto, as moléculas com alta persistência (baixa taxa de degradação), como os compostos organoclorados, podem permanecer no ambiente sem sofrer qualquer alteração. Estas moléculas podem ser adsorvidas nas partículas do solo e carreadas pelas águas superficiais (SANCHES et al., 2003).

A cinética dos herbicidas no ambiente permite visualizar o quanto interligado e dinâmico é o transporte destas substâncias entre os diferentes compartimentos ambientais. $\mathrm{O}$ ambiente aquático, no entanto, é o principal escoadouro dos defensivos agrícolas. A contaminação das águas superficiais tem sido documentada em todo o mundo e constitui uma das maiores preocupações ambientais no que diz respeito à escala local, regional, nacional e global (KONSTANTINOU et al., 2006).

Adeterminação dos herbicidas com probabilidade de atingirem às águas subterrâneas e superficiais é normalmente efetuada com base em índices de particionamento, considerando as propriedades físico-químicas e persistência no ambiente, como índice de GUS, métodos de GOSS e critérios da EPA (GUSTAFSON, 1989; SANCHES et al., 2003). Porém, o alto custo das análises químicas para o monitoramento constante de todos os pesticidas empregados ou daqueles prováveis de atingirem os recursos hídricos requer uma identificação do uso nas áreas em estudo, de modo a se atingir eficiência econômica e técnica no monitoramento (ARMAS et al., 2005).

A presença de qualquer componente químico em concentrações acima dos especificados nas normas padrão, pode provocar efeitos tóxicos, seja na saúde humana ou no ambiente (YOUNES; GALAL-GORCHEV, 2000). Esse fato desperta o interesse em verificar se há a ocorrência de contaminação dos ambientes aquáticos (DORES; DE-LAMONICA-FREIRE, 2001).

Aproximadamente $27 \%$ do abastecimento de água da cidade de Aracaju, capital do Estado de Sergipe, é proveniente da Sub-bacia do Rio Poxim, cujas áreas de recarga, antes ocupadas por vegetação nativa, vêm sendo substituídas pela atividade agropecuária, principalmente pelo cultivo da cana-de-açúcar. A redução da vazão do rio e o aumento da poluição na sub-bacia têm comprometido a oferta hídrica e a qualidade da água (SILVA et al., 2004).

O objetivo desse estudo foi avaliar a contaminação da água superficial no alto curso do Rio Poxim com herbicidas utilizados na cultura da cana-de-açúcar. Esse diagnóstico é importante para o direcionamento de melhores condições de manejo da cultura na Sub-bacia, podendo servir de referência para regiões similares produtoras do Estado de Sergipe.

\section{Material e métodos}

\section{Área de Estudo}

A Sub-bacia hidrográfica do Rio Poxim está situada entre as coordenadas geográficas $11^{\circ} 01^{\prime}$ e $10^{\circ} 47^{\prime}$ de latitude Sul e $37^{\circ} 01^{\prime}$ e $37^{\circ} 24^{\prime}$ de longitude Oeste, inserida na Região Metropolitana de Aracaju. O principal rio da Sub-bacia hidrográfica em estudo é o Rio Poxim e tem como afluentes os rios Poxim-Mirim e Poxim-Açu. A Sub-bacia do rio Poxim (FIG. 1), ocupa uma área de $460 \mathrm{~km}^{2}$ e faz parte da bacia hidrográfica do rio Sergipe, que drena uma superfície de $3.670 \mathrm{~km}^{2}$, a qual é considerada a mais importante do Estado, em termos de número de habitantes, de demanda de água e de concentração de indústrias (ANA, 2001).

As condições climáticas que predominam nessa região são: o clima tropical úmido, com período seco de setembro a março e período chuvoso de abril a agosto, com precipitação pluvial média anual variando de 1.600 e $1.900 \mathrm{~mm}$. A temperatura média do ambiente é de $23^{\circ} \mathrm{C}$, para os meses mais frios, junho a agosto, e de $31^{\circ} \mathrm{C}$ para os meses mais quentes, dezembro a fevereiro (SOARES, 2001).

\section{Avaliação dos riscos de contaminação dos herbicidas nas águas subterrâneas e superficiais}

As informações dos principais produtos comerciais aplicados na região foram obtidas durante visitas as principais propriedades rurais produtora de cana-de-açúcar na Sub-bacia do Rio Poxim, e estabelecimentos comerciais de venda de herbicidas na cidade de Aracaju. Foram catalogados os herbicidas 
Figura 1 - Limites e estações de amostragem de água na Subbacia hidrográfica do Rio Poxim

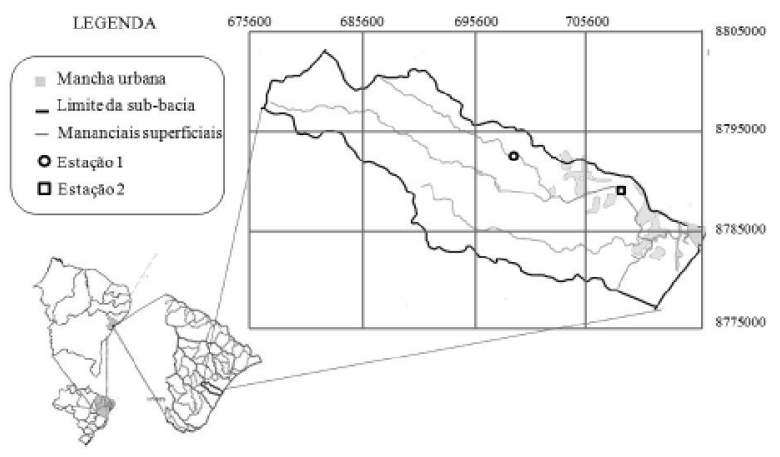

Fonte: Sergipe (2004)

mais comumente usados e vendidos na região. Com estes dados buscou-se identificar as características e propriedades físico-químicas dos herbicidas por meio de pesquisa na Agência Nacional de Vigilância Sanitária (ANVISA) e no banco de dados Pesticide Properties Data Base (PPDB, 2010; EXTOXNET, 2010).

Para a análise de riscos utilizou-se o índice de Groundwater Ubiquity Score (GUS), os critérios da Environmental Protection Agency (EPA) e o método GOSS. Esses métodos permitem avaliar a capacidade de provável risco de contaminação das águas subterrâneas e superficiais, por meio de informações sobre os princípios ativos.

\section{Índice Groundwater ubiquity score (GUS)}

O índice proposto por Groundwater Ubiquity Score avalia o potencial de contaminação de água subterrânea por herbicida segundo a equação 1 .

GUS $=\log \left(\mathrm{t}_{\text {solo }}^{1 / 2}\right) \times(4-\log ($ Koc $))$

onde:

$\mathrm{t}^{1 / 2}$ solo $=$ meia vida do produto no solo (dias);

Koc $=$ coeficiente de adsorção ao carbono orgânico $\left(\mathrm{L} \mathrm{kg}^{-1}\right)$.

Após a obtenção do valor do índice de GUS, o principio ativo é classificado em uma das categorias, definidas por faixas pré-estabelecidas, conforme os seguintes intervalos:

a) GUS $\leq 1,8$ => Não sofre lixiviação;

b) $1,8<$ GUS $<2,8=>$ Faixa de Transição;

c) GUS $\geq 2,8$ => Provável Lixiviação.

\section{Critérios da Environmental Protection Agency (EPA)}

Outra forma utilizada para avaliar a capacidade de transporte de herbicidas, é o critério da (EPA) (COHEN et al., 1995). Os princípios ativos que obedecerem às condições abaixo oferecem maior potencial de risco de transporte e, consequente tendência à contaminação, principalmente de águas subterrâneas:

a) solubilidade em água $>30 \mathrm{mg} \mathrm{L}^{-1}$;

b) coeficiente de adsorção à matéria orgânica: Koc < 300 a $500 \mathrm{~mL} \mathrm{~g}^{-1}$;

c) constante de Henry: $\mathrm{kH}<10^{-2} \mathrm{~Pa} \mathrm{~m}^{3} \mathrm{~mol}^{-1}$;

d) meia vida no solo ( $\mathrm{t}^{1} \frac{2}{2}$ solo): > 14 a 21 dias;

e) meia vida na água ( $\mathrm{t}^{1} \frac{2}{2}$ água $)>175$ dias.

\section{Método de Goss}

O método proposto por GOSS (1992), utiliza um conjunto de cláusulas e regras, apresentadas em intervalos matemáticos pelos quais se faz a avaliação do potencial de transporte de herbicidas associado a sedimento ou dissolvido em água superficiais (TAB. 1).

As substâncias que não se enquadram em nenhum dos critérios acima são consideradas com médio potencial para contaminarem águas superficiais. Para calcular os valores do índice de GUS, critérios EPAe método de GOSS foi utilizado o programa AGROSCRE da Embrapa que faz a avaliação de tendências de transporte de princípios ativos de herbicidas mediante o fornecimento dos dados físico-químicos de cada princípio ativo.

Tabela 1 - Método proposto por GOSS

A) Potencial de transporte associado ao sedimento $\mathrm{t}^{1 / 2}$ solo $(\mathrm{d}) \quad \operatorname{Koc}\left(\mathrm{mL} \mathrm{g}^{-1}\right) \quad \mathrm{Ws}\left(\mathrm{mg} \mathrm{L}^{-1}\right)$

$\begin{array}{lccc} & \geq 40 & \geq 1000 & - \\ \text { Alto Potencial } & \geq 40 & \geq 500 & \leq 0,5 \\ & <1 & - & - \\ & \leq 2 & \leq 500 & - \\ \text { Baixo Potencial } & \leq 4 & \leq 900 & \geq 0,5 \\ & \leq 40 & \leq 500 & \geq 0,5 \\ & \leq 40 & \leq 900 & \geq 2\end{array}$

B) Potencial de transporte dissolvido em água $\mathrm{t}^{1} \frac{2}{2}$ solo $(\mathrm{d}) \quad \operatorname{Koc}\left(\mathrm{mL} \mathrm{g}^{-1}\right) \quad \mathrm{Ws}\left(\mathrm{mg} \mathrm{L}^{-1}\right)$

Alto Potencial

$>35<100000 \geq 1$

$<35 \quad \leq 700 \quad \geq 10 \mathrm{e} \leq 100$

$-\quad \geq 100000 \quad-$

Baixo Potencial $\leq 1 \leq 1000$

$$
<35
$$$$
<0,5
$$

t²: meia-vida no solo (dias); Koc: coeficiente de absorção de matéria orgânica $\left(\mathrm{mL} \mathrm{g}^{-1}\right)$; Ws: solubilidade em água $\left(\mathrm{mg} \mathrm{L}^{-1}\right)$. Fonte: (Milhome et al., 2009) 


\section{Monitoramento dos herbicidas Diuron e Ametrina na Sub-bacia do Rio Poxim}

Realizada a avaliação dos ricos de contaminação por herbicidas das águas subterrâneas e superficiais, foram definidos pontos de amostragem de água em duas estações de monitoramento, distribuídas ao longo da Subbacia hidrográfica do Rio Poxim, para identificar possíveis contaminantes. O primeiro ponto foi definido a montante da área ocupada por plantações de cana-de-açúcar no Rio Poxim-Mirim, coordenadas UTM 24L 699279, 8794697; o segundo ponto na área de captação da DESO coordenadas UTM 24L 707949, 8791936 no Rio Poxim. Esse ponto foi determinado para verificar a presença de herbicidas na área de captação de água para abastecimento público da cidade de Aracaju.

As amostras de água foram coletadas no período de julho de 2009 a julho de 2010, em dois pontos de monitoramento por meio de coletas bi-mensais, totalizando 14 amostras. Com isso, foi possível avaliar a qualidade da água nos períodos chuvoso e seco. As amostras de água foram levadas a laboratório e analisadas pelo método Multiresíduos utilizando extração líquidolíquido. A quantificação foi realizada no cromatógrafo a gás, com detectores de captura de elétrons e cromatógrafo liquido. Os dados obtidos no monitoramento foram tabulados e analisados por meio de estatística descritiva.
Os resultados foram representados em tabelas e gráficos do tipo séries temporais.

\section{Resultados e discussões}

\section{Risco de contaminação de águas subterrâneas e superficiais}

O levantamento dos herbicidas usados no alto da Sub-bacia Rio Poxim resultou em uma lista de 14 princípios ativos mais comumente utilizados (TAB. 2) formulados isoladamente ou em misturas, totalizando 34 marcas comerciais, distribuídos em 14 grupos químicos, sendo o grupo das triazinas e sulfonilureia representado por dois produtos, enquanto os demais compreendem uma molécula. Ao longo dos anos, o uso de herbicidas a base de ureia e triazianas na cana-de-açúcar, tem tido grande expressão. Todos os princípios ativos e marcas comerciais empregados encontram-se registrados para a cultura da cana-de-açúcar, seja no Ministério da Agricultura, Pecuária e Abastecimento, seja na Secretaria de Agricultura e Abastecimento do Estado de Sergipe.

$\mathrm{Na}$ Tabela 3 são apresentados os resultados da análise do potencial de contaminação de águas subterrâneas, pelos princípios ativos mais comumente utilizados na região, conforme os critérios de "screening", sugeridos

Tabela 2 - Propriedades físico-químicas dos princípios ativos dos herbicidas, a 20-25 ${ }^{\circ} \mathrm{C}$, usados na região da Sub-Bacia do Rio Poxim

\begin{tabular}{|c|c|c|c|c|c|c|c|c|c|}
\hline \multirow{2}{*}{ Princípio ativo* } & \multirow{2}{*}{ Grupo Químico } & \multirow{2}{*}{$\mathrm{Uso}^{\mathrm{a}}$} & $\mathrm{T}^{1 / 2}$ & $\mathrm{~T}^{1 / 2}$ & \multirow{2}{*}{$\mathrm{Koc}^{\mathrm{d}}$} & \multirow{2}{*}{$\mathrm{Ws}^{\mathrm{e}}$} & \multirow{2}{*}{$\mathrm{Vp}^{\mathrm{f}}$} & \multirow{2}{*}{$\mathrm{KH}^{\mathrm{g}}$} & \multirow{2}{*}{$\frac{\text { Clas }}{\text { toxic }^{h}}$} \\
\hline & & & solo $^{b}$ & água ${ }^{c}$ & & & & & \\
\hline 1 - metribuzim & triazinona & $\mathrm{R} / \mathrm{O}$ & 140 & 4760 & 106 & $1,03.103$ & $5,80.10-5$ & $1,43.10-9$ & IV \\
\hline 2 - diuron & ureia & $\mathrm{R} / \mathrm{O}$ & 372 & 1290 & 499 & $3,60.101$ & $9,20.10-6$ & $5,1.10-5$ & III \\
\hline 3 - ametrina & triazina & $\mathrm{R} / \mathrm{O}$ & 37 & 28 & 245 & $2,04.102$ & $3,65.10-4$ & $4,14.10-4$ & III \\
\hline 4 - atrazina & triazina & $\mathrm{R} / \mathrm{O}$ & 146 & 30 & 93 & $3,30.101$ & $4,00.10-5$ & $2,48.10-4$ & III \\
\hline 5 - glifosato & glicina substituída & $\mathrm{O} / \mathrm{M}$ & 96 & 35 & 6922 & $1,16.104$ & $5,73.10-8$ & $1,4.10-7$ & III \\
\hline 6 - paraquat & bipiridílio & $\mathrm{O}$ & 620 & 30 & 1.0 .104 & $6,26.105$ & $1,35.10-5$ & - & I \\
\hline 7 - hexazinona & triazinona & $\mathrm{R} / \mathrm{O}$ & 222 & 56 & 640 & $2,9.104$ & $3,00.10-5$ & - & III \\
\hline 8 - trifloxissulfurom & sulfonilureia & $\mathrm{O}$ & 52 & - & 80 & $5,02.103$ & $1,00.10-7$ & - & III \\
\hline 9 - halossulfurom & sulfonilureia & $\mathrm{O}$ & 51 & 14 & 124 & $1,65.103$ & $1,33.10-5$ & $1,1.10-3$ & III \\
\hline 10 - oxifluorfem & éter difenílico & $\mathrm{R} / \mathrm{O}$ & 30 & - & 1.0 .105 & $1,0.10-1$ & $2,6.10-5$ & - & II \\
\hline $11-2,4-\mathrm{D}$ & ácido Ariloxialcanóico & $\mathrm{R} / \mathrm{O}$ & 34 & 39 & 46 & $2,76.104$ & $1,10.10-2$ & $1,8.10-7$ & I \\
\hline 12 - picloram & ácido Piridinocarboxílico & $\mathrm{R} / \mathrm{O}$ & 324,7 & - & 16 & $4,30.102$ & $9,61.10-9$ & $4,7.10-5$ & II \\
\hline 13 - MSMA & organoarsênico & $\mathrm{O}$ & 269 & 35 & 1680 & $1,04.106$ & $3,50.101$ & - & III \\
\hline 14 - imazapir & imidazolinona & $\mathrm{R} / \mathrm{O}$ & 1200 & 30 & 81 & - & - & $3,0.10-7$ & III \\
\hline
\end{tabular}

Nomenclatura de acordo com as regras brasileiras*; “_“ valor não encontrado na literatura ou não calculado por falta de parâmetros; a = usos na cultura da cana-de-açúcar: R - Pré-emergente; O - Pós-emergente; M - Maturador; b = meia-vida no solo, em dias; c = meia-vida na água, em dias; $d$ = coeficiente de adsorção normalizado pela fração de carbono orgânico do solo (L kg-1); e = solubilidade em água (mg L-1); f = pressão de vapor, em MPa., g = constante de Henry kH.; h = classe toxicológica (I - extremamente tóxico; II - altamente tóxico; III - medianamente tóxico; IV - pouco tóxico). Dados extraídos de: (PPDB, 2010 e Extoxnet, 2010) 
Tabela 3 - Avaliação de risco de contaminação de águas subterrâneas, pelo índice de GUS, e critérios da EPA

\begin{tabular}{llccc}
\hline \multicolumn{1}{c}{ Princípio ativo } & \multicolumn{1}{c}{ Grupo Químico } & GUS & EPA \\
\hline metribuzim & triazinona & $\mathrm{L}$ & 4,24 & $\mathrm{~L}$ \\
diuron & ureia & $\mathrm{L}$ & 3,35 & $\mathrm{~L}$ \\
ametrina & triazina & $\mathrm{T}$ & 2,53 & $\mathrm{NL}$ \\
atrazina & triazina & $\mathrm{L}$ & 4,40 & $\mathrm{NL}$ \\
glifosato & glicina substituída & $\mathrm{NL}$ & 0,32 & $\mathrm{NL}$ \\
paraquat & bipiridílio & $\mathrm{NL}$ & 0,00 & $\mathrm{NA}$ \\
hexazinona & triazinona & $\mathrm{L}$ & 2,80 & $\mathrm{NA}$ \\
trifloxissulfurom-sódico & t sulfonilureia & $\mathrm{L}$ & 3,60 & $\mathrm{NA}$ \\
halossulfurom-metílico & sulfonilureia & $\mathrm{L}$ & 3,26 & $\mathrm{NL}$ \\
oxifluorfem & éter difenílico & $\mathrm{NL}$ & 1,48 & $\mathrm{NA}$ \\
2,4-d & ácido ariloxialcanóico & $\mathrm{L}$ & 3,58 & $\mathrm{NL}$ \\
picloram & ácido piridinocarboxílico & $\mathrm{L}$ & 7,02 & $\mathrm{NL}$ \\
msma & organoarsênico & $\mathrm{T}$ & 1,88 & $\mathrm{NA}$ \\
imazapir & imidazolinona & $\mathrm{L}$ & 6,44 & $\mathrm{NA}$ \\
\hline
\end{tabular}

Resultados fornecidos pelo programa AGROSCRE, GUS = Índice do potencial de lixiviação, onde L= Provável lixiviação; NL = Não lixívia; $\mathrm{T}$ = Faixa de transição; $\mathrm{EPA}=$ avaliação pelo critério da EPA (onde NA= Não avaliado por falta de informações; $\mathrm{L}=$ Provável lixiviação, NL = Não sofre lixiviação)

pela EPA e pelo índice de GUS. Princípios ativos como paraquat, hexazinona, trifloxissulfurom-sódico, imazapir e MSMA não puderam ser avaliados pelos critérios da EPA por falta das informações como: constante de Henry $\left(\mathrm{k}_{\mathrm{H}}\right)$, pressão de vapor $(\mathrm{Vp})$, tempo de meia-vida na água $\left(\mathrm{T}_{1 / 2 \text { g gua }}\right)$ e solubilidade em água $(\mathrm{Ws})$.

Utilizando os critérios da EPA, na (TAB. 3), pode-se dizer que: os herbicidas metribuzim e diuron apresentaram considerável risco de contaminação nas águas subterrâneas. Por outro lado, os demais herbicidas não mostraram tendência para contaminação dessas águas.

A persistência de um composto no ambiente é normalmente medida pela meia-vida, definida como o tempo necessário para que ocorra a dissipação de $50 \%$ da quantidade inicial do herbicida aplicado (SILVA et al., 2007). O tempo de meia-vida na água para o metribuzim e diuron comparados com outros princípios ativos foi bastante elevado, ou seja, este critério pode ajudar a prever o tempo de permanência e o provável risco de contaminação dos lençóis freáticos subterrâneos antes mesmo da aplicação do herbicida.

Pelo índice de GUS, que analisa o tempo de meia-vida do solo $\left(\mathrm{T}_{1 / 2 \text { solo }}\right)$ e coeficiente de adsorção ao carbono orgânico (Koc), verificou-se que o metribuzim, diuron, atrazina, hexazinona, trifloxissulfurom-Sódico, halossulfurom-metílico, 2,4-D, picloram, e imazapir apresentaram tendência para lixiviação (TAB. 3).
Os métodos utilizados neste trabalho apresentam características de análise diferenciadas, ou seja, ao se comparar o GUS com o EPA, observa-se que quando o Koc apresenta valores baixos, o princípio ativo sofre provável lixiviação em decorrência da capacidade do mesmo adsorver a matéria orgânica.

Figura 2 - Resultados da avaliação de risco de contaminação de águas subterrâneas com base no índice de GUS, na Sub-Bacia do Rio Poxim

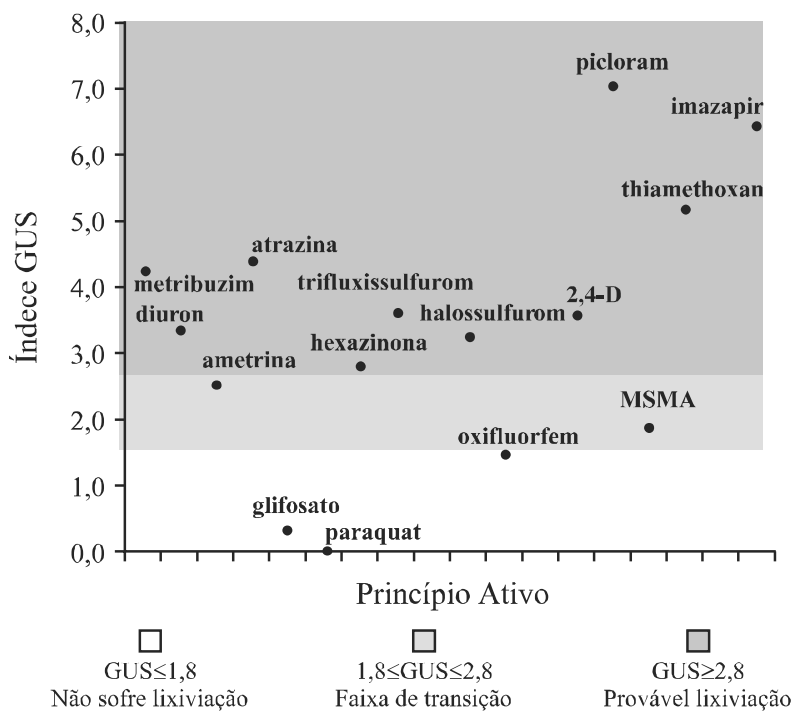


Os resultados da Figura 2 indicam que, de acordo com o índice de GUS, entre os 14 princípios ativos calculados existem 9, que sofrem prováveis riscos de lixiviação (GUS > 2,8), sendo que a o Imazapir e Picloram apresentaram os maiores valores para o índice de GUS, 6,44 e 7,02, respectivamente. Esses resultados mostram a possibilidade dessas moléculas sofrerem lixiviação, podendo atingir águas subterrâneas. Resultados semelhantes foram encontrados na Bacia do Rio Corumbataí/SP e em Primavera do Leste/MT (DORES; DE-LAMONICA-FREIRE, 2001; ARMAS et al., 2005). Apenas três princípios ativos não expressaram perigo (GUS < 1,8) por não sofrerem lixiviação no perfil do solo são eles: o Glifosato, Paraquat e Oxifluorfem. Já o MSMA e Ametrina apresentaram valores na faixa de transição $(1,8 \leq \mathrm{GUS} \geq 2,8)$ podendo ou não sofrer lixiviação.

De acordo com o risco de contaminação da água superficial, os herbicidas foram divididos entre os que podem ser transportados dissolvidos em água, e aqueles que são transportados associados ao sedimento em suspensão.

A classificação, segundo o método de GOSS, para os herbicidas em estudo é apresentada na (TAB. 4). Dentre aqueles com alto potencial de transporte associado ao sedimento, estão: Paraquat, Glifosato, MSMA. Os herbicidas que apresentaram maior capacidade de mobilidade quando disperso em água, são: Metribuzim; Diuron; Ametrina; Atrazina; Glifosato; Paraquat; Hexazinona; Trifloxissulfurom-Sódico; Halossulfurom-
Metílico, Diuron e Glifosato, sendo que destes o Diuron também é contaminante em potencial de águas subterrâneas.

\section{Resultados do monitoramento dos herbicidas Diuron e Ametrina}

Foi identificada a presença do princípio ativo Diuron, nos rios Poxim-Mirim e Poxim, em níveis que podem comprometer a qualidade da água. A presença desse herbicida no Rio Poxim-Mirim ocorreram nos meses de julho, setembro e novembro de 2009, e março, maio e julho de 2010, enquanto que no Rio Poxim, ocorreram nos meses de maio e julho de 2010. As demais amostras apresentaram resultados abaixo do limite de quantificação do método: $0,03 \mu \mathrm{g} \mathrm{L}^{-1}$ para organohalogenados e $0,01 \mu \mathrm{g} \mathrm{L}^{-1}$ para organofosforados (TAB. 5).

Os valores encontrados para diuron nos meses de julho de 2009, maio e julho de 2010 com concentrações de 0,$2 ; 0,9$ e $0,2 \mu \mathrm{g} \mathrm{L}^{-1}$, respectivamente são preocupantes quando comparados com padrões da Comunidade Européia. Esta admite concentração máxima de $0,1 \mu \mathrm{g} \mathrm{L} \mathrm{L}^{-1}$ para qualquer pesticida em águas destinadas para consumo humano e em $0,5 \mu \mathrm{g} \mathrm{L}^{-1}$ para o total de resíduos, sem deixar claro se deve, ou não, considerar também produtos de transformação (ARMAS et al., 2007; FILIZOLA et al., 2002;). Este limite tem sido motivo de questionamento uma vez que não considera a toxicidade de cada produto, e ainda, as metodologias analíticas disponíveis para alguns compostos que não atingem limites de detecção desta ordem de

Tabela 4 - Classificação dos princípios ativos pelo método GOSS obtido com o programa AGROSCRE

\begin{tabular}{lccc}
\hline \multicolumn{1}{c}{ Principio ativo } & Grupo químico & & GOSS \\
\cline { 2 - 3 } metribuzim & triazinona & Sedimento & Dissolvido \\
diuron & ureia & $\mathrm{M}$ & $\mathrm{A}$ \\
ametrina & triazina & $\mathrm{M}$ & $\mathrm{A}$ \\
atrazina & triazina & $\mathrm{B}$ & $\mathrm{A}$ \\
glifosato & glicina substituída & $\mathrm{M}$ & $\mathrm{A}$ \\
paraquat & bipiridílio & $\mathrm{A}$ & $\mathrm{A}$ \\
hexazinona & triazinona & $\mathrm{A}$ & $\mathrm{A}$ \\
trifloxissulfurom-sódico & t sulfonilureia & $\mathrm{M}$ & $\mathrm{A}$ \\
halossulfurom-metílico & Sulfonilureia & $\mathrm{M}$ & $\mathrm{A}$ \\
oxifluorfem & éter difenílico & $\mathrm{M}$ & $\mathrm{A}$ \\
$2,4-\mathrm{d}$ & ácido ariloxialcanóico & $\mathrm{M}$ & $\mathrm{B}$ \\
picloram & ácido piridinocarboxílico & $\mathrm{B}$ & $\mathrm{M}$ \\
msma & organoarsênico & $\mathrm{M}$ & $\mathrm{A}$ \\
imazapir & midazolinona & $\mathrm{A}$ & $\mathrm{A}$ \\
\hline $\mathrm{A}$ & $\mathrm{NA}$ & $\mathrm{NA}$ \\
\hline
\end{tabular}

A - Alto potencial de transporte; B - baixo potencial de transporte; M - médio potencial de transporte e NA - não analisado 
Tabela 5 - Concentração do diuron e ametrina, no período chuvoso e seco, no alto da Sub-Bacia do Rio Poxim

\begin{tabular}{lcccccc}
\hline \multirow{2}{*}{ Data da Coleta } & \multirow{2}{*}{ Precipitação pluvial } & \multirow{2}{*}{ Período } & \multicolumn{2}{c}{ Rio Poxim-Mirim } & \multicolumn{2}{c}{ Rio Poxim (captação) } \\
\cline { 3 - 6 } & & (diuron) & (ametrina) & (diuron) & (ametrina) \\
\hline $29 / 07 / 2009$ & 220,3 & Chuvoso & $0,2 \mu \mathrm{gL}^{-1}$ & $0,5 \mu \cdot \mathrm{gL}^{-1}$ & $<\mathrm{LD}$ & $<\mathrm{LD}$ \\
$23 / 09 / 2009$ & 122,6 & Seco & $0,1 \mu \mathrm{gL}^{-1}$ & $0,03 \mu \cdot \mathrm{gL}^{-1}$ & $<\mathrm{LD}$ & $<\mathrm{LD}$ \\
$27 / 11 / 2009$ & 0,0 & Seco & $0,03 \mu \mathrm{gL}^{-1}$ & $<\mathrm{LD}$ & $<\mathrm{LD}$ & $<\mathrm{LD}$ \\
$20 / 01 / 2010$ & 88,6 & Seco & $<\mathrm{LD}$ & $<\mathrm{LD}$ & $<\mathrm{LD}$ & $<\mathrm{LD}$ \\
$31 / 03 / 2010$ & 31,0 & Seco & $0,1 \mu \mathrm{gL}^{-1}$ & $<\mathrm{LD}$ & $<\mathrm{LD}$ & $<\mathrm{LD}$ \\
$25 / 05 / 2010$ & 219,2 & Chuvoso & $0,9 \mu \mathrm{gL}^{-1}$ & $<\mathrm{LD}$ & $0,04 \mu \mathrm{gL}^{-1}$ & $<\mathrm{LD}$ \\
$28 / 07 / 2010$ & 235,9 & Chuvoso & $0,2 \mu \mathrm{gL}^{-1}$ & $<\mathrm{LD}$ & $0,05 \mu \mathrm{gL}^{-1}$ & $<\mathrm{LD}$ \\
\hline
\end{tabular}

Nota: <LD - Valor abaixo do Limite de Detecção do método

grandeza (WALLS et al., 1996). Por outro lado, a EPA e a Organização Mundial da Saúde estabelecem níveis máximos para pesticidas individuais em água destinada ao consumo humano, baseados em estudos toxicológicos e epidemiológicos.

Para Watts et al. (2000), picos de concentrações em águas superficiais são registrados logo após eventos de chuva de alta intensidade, especialmente, quando as áreas ao redor dos mananciais tenham sido recentemente tratadas com altas doses de pesticidas. Mesmo em concentrações baixas, os pesticidas representam riscos à saúde humana (YOUNES; GALAL-GORCHEV, 2000). Não existe nível seguro previsível para pesticidas em água, quando pode ocorrer biomagnificação (DORES; DE-LAMONICA-FREIRE, 2001).

Pode-se observar na Tabela 5 que as concentrações do pesticida diuron no Rio Poxim-Mirim, estão relacionadas com a precipitação pluvial. Nesse período ocorreram chuvas que influenciaram o carreamento do herbicida, tanto para o Rio Poxim-Mirim (FIG. 3A) quanto para o Rio Poxim (FIG. 3B).

Matallo et al. (2005) determinaram que 52\% da quantidade aplicada de um herbicida usado na cultura de cana-de-açúcar no Brasil lixiviou abaixo de $50 \mathrm{~cm}$ em um solo arenoso, durante um ano. Barriuso et al. (1996), citaram que cerca de $20 \%$ das quantidades dos pesticidas usados podem alcançar as águas superficiais e que esse número só não é maior porque existem alguns processos que atuam na imobilização de moléculas de pesticidas, diminuindo a quantidade de poluentes.

A transferência de moléculas de herbicidas dos ecossistemas terrestres aos aquáticos é uma constante, sobretudo em áreas agrícolas, devido ao uso de elevadas quantidades e de diferentes tipos de princípios ativos por área e às altas taxas de erosão do solo (BORTOLUZZI et al., 2006).
Figura 3 - Relação entre a precipitação pluvial e a concentração do Diuron, entre o período de julho de 2009 a julho de 2010, no Rio Poxim Mirim (A) e Rio Poxim (B)
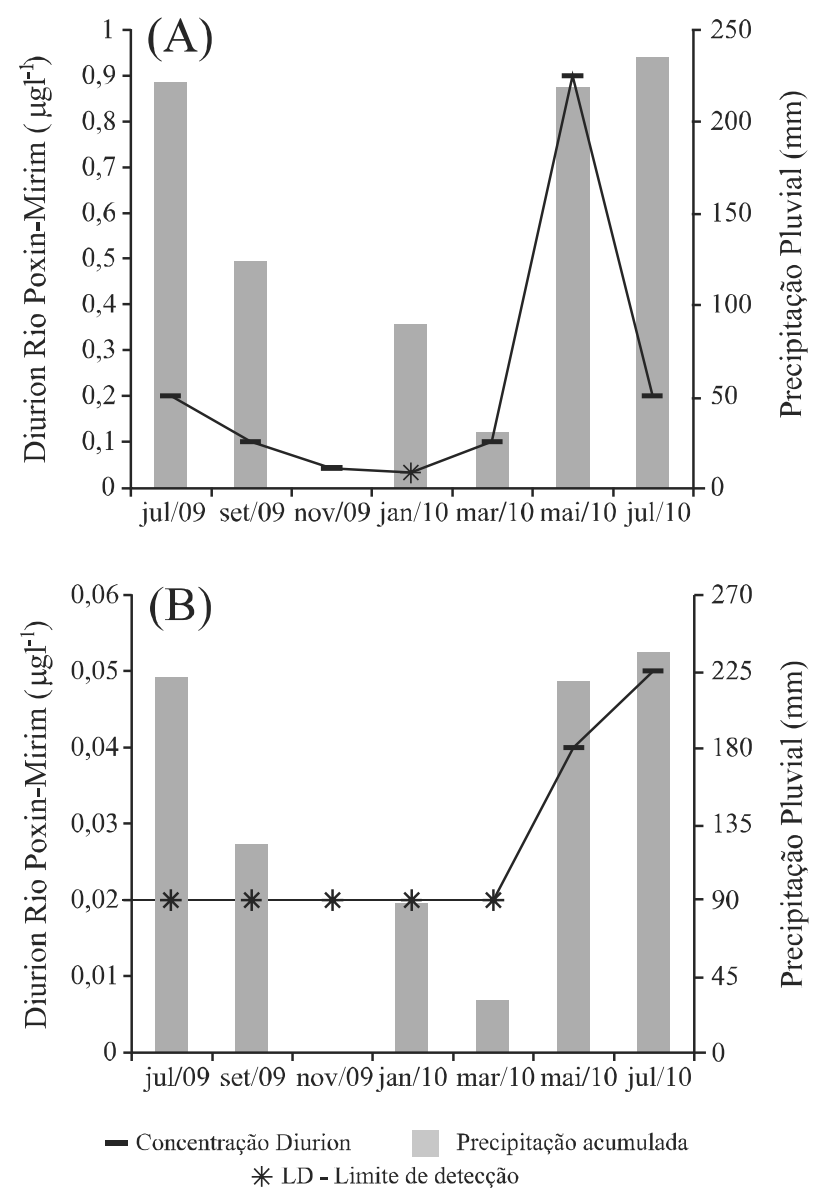

Conforme procedimento de manejo os pesticidas são aplicados em dias preferencialmente secos e ficam retidos nas plantas e no solo. Quando ocorrem as chuvas, 
esses são carreados por meio do escoamento superficial ou percolação em direção às águas superficiais e subterrâneas.

Figura 4 - Relação entre a precipitação pluvial e a concentração do ametrina, entre o período de julho de 2009 a julho de 2010, no Rio Poxim-Mirim

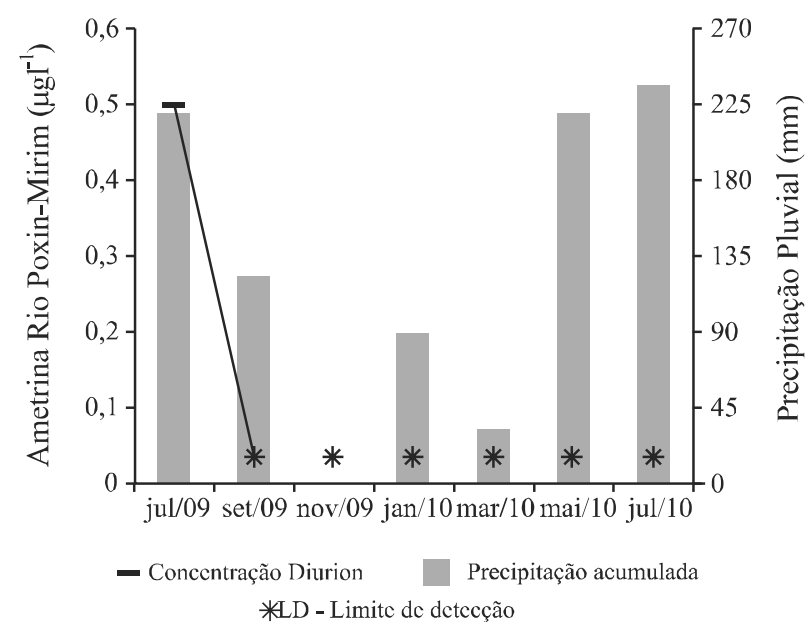

Quanto à ametrina verificou-se a presença apenas no Rio Poxim-Mirim. A detecção ocorreu nos meses de julho e setembro 2009. Nos demais meses de coleta os limites ficaram abaixo da quantificação do método (FIG. 4).

A simples detecção de resíduos de herbicida é preocupante, pois em termos ecológicos o efeito crônico (ao longo do tempo) da contaminação, mesmo sob concentrações baixas (sub-letais), pode originar alterações imperceptíveis em longo prazo, com a diminuição do potencial biológico (diminuição do sucesso reprodutivo ou maior suscetibilidade a doenças, por exemplo) de espécies tanto animais quanto vegetais (CALHEIROS et al., 2006).

Sendo razoavelmente estável, a ametrina apresenta uma meia-vida de 20 a 100 dias, é muito móvel no ambiente e ainda altamente persistente em água e solo. Seus resíduos e metabólitos têm sido encontrados em água subterrânea, mesmoapóslongos períodos desuaaplicação(IGNJATOVIC et al., 1998). A pouca frequência da ametrina na água pode ter sido uma consequência deste princípio ativo estar abaixo dos limites de detecção do método analítico ou mesmo a não mais utilização por parte dos produtores.

Estes resultados indicam que o manejo de herbicidas na cana-de-açúcar influencia a qualidade da água da subbacia do Rio Pixim e alertam para a realização de estudos mais detalhados que possam identificar outros princípios ativos, como também demonstram a importância do monitoramento da qualidade da água para dar suporte à avaliação de risco de contaminação dos usuários.

\section{Conclusões}

1. Foram detectadas concentrações de diuron e ametrina acima dos padrões internacionais para água destinada ao consumo humano no Rio Poxim-Mirim;

2. As concentrações foram maiores no período chuvoso em decorrência da época de aplicação na cultura e do maior escoamento superficial do solo;

3. As análises de risco de contaminação de herbicidas mostraram que o princípio ativo diuron apresenta média capacidade de transporte no sedimento, elevada capacidade de transporte na água, alta lixiviação e elevado risco de contaminação de água subterrânea. A ametrina apresenta elevada capacidade de transporte na água e médio potencial de lixiviação;

4. A concentração de diuron $\left(0,05 \mu \mathrm{g} \mathrm{L}^{-1}\right)$ encontradas no Rio Poxim na captação para abastecimento público evidencia um grande alerta a população, visto que os tratamentos convencionais de água não são capazes de retirar essa substância da água.

\section{Agradecimentos}

À Fundação de Amparo à Pesquisa e à Inovação Tecnológica do Estado de Sergipe (FAPITEC), pelo apoio no financiamento do projeto de pesquisa.

\section{Referencias}

AGÊNCIA NACIONAL DAS ÁGUAS (ANA). A gestão dos recursos hídricos no Estado de Sergipe. Sergipe, 2001. (Sistema nacional de informações sobre recursos hídricos, CD-ROM, 1).

ARMAS, E. D. et al. Diagnóstico espaço-temporal da ocorrência de herbicidas nas águas superficiais e sedimentos do Rio Corumbataí e principais afluentes. Química Nova, v. 30, n. 05, p. 1119-1127, 2007.

ARMAS, E. D. et al. Uso de agrotóxicos em cana-de-açúcar na bacia do rio Corumbataí e o risco de poluição hídrica. Química Nova, v. 28, n. 06, p. 975-982, 2005.

BARRIUSO, E. et al. Les pesticides et les polluants organiques des sols: transformations et dissipation. Étude et Gestion des Sols, v. 03, n. 04, p. 279-296, 1996.

BORTOLUZZI, E. C. et al. Contaminação de águas superficiais por agrotóxicos em função do uso do solo numa microbacia 
hidrográfica de Agudo, RS. Revista Brasileira de Engenharia Agrícola e Ambiental, v. 10, n. 04, p. 881-887, 2006.

BRITO, N. M. et al. Risco de contaminação de águas por pesticidas aplicados em plantações de eucaliptos e coqueiros: análise preliminar. Pesticidas: Revista de Ecotoxicologia e Meio Ambiente, v. 11, p. 93-104, 2001.

CALHEIROS, D. F.; OLIVEIRA, M. D.; DOLORES, E. F. G. Poluição por pesticidas, nutrientes e material em suspensão nos rios formadores do Pantanal Matogrossense. Corumbá, MS: Embrapa Pantanal, 2006. 4 p. (ADM - Artigo de Divulgação na Mídia, 096). Disponível em: <http://www.cpap.embrapa.br/ publicacoes/ online/ADM096>. Acesso em: 10 mar. 2011.

COHEN, S. Z. et al. Offsite transport of pesticides in water mathematical models of pesticide leaching and runoff. Pure and Applied Chemistry, v. 67, n. 12, p. 2109-2148, 1995.

DORES, E. F. G. C.; DE-LAMONICA-FREIRE, E. M. Contaminação do ambiente aquático por pesticidas. Estudo de caso: Águas usadas para consumo humano em Primavera do Leste, Mato Grosso - Análise preliminar. Química Nova, v. 24, n. 01, p. 27-36. 2001.

EXTENSION TOXICOLOGY NETWORK (EXTOXNET). Pesticides active ingredient profiles. 2010. Disponível em: <http:// pmep.cce.cornell.edu/profiles/extoxnet>. Acesso em: 15 abr. 2010.

FILIZOLA, H. F. et al. Monitoramento e avaliação do risco de contaminação por pesticidas em água superficial e subterrânea na região de Guaíra. Pesquisa Agropecuária Brasileira, v. 37, n. 05, p. 659-667, 2002.

GOMES, M. A. F.; SPADOTTO, C. A.; LANCHOTTE, V. L. Ocorrência do herbicida Tebutiuron na água subterrânea da microbacia do Córrego do Espraiado, Ribeirão Preto - SP. Pesticidas: Revista de Ecotoxicologia e Meio Ambiente, v. 11, p. 65-76, 2001.

GOSS, D. W. Screening procedure for soils and pesticides for potential water quality impacts. Weed Technology, v. 06, n. 03, p. 701-708, 1992.

GRÜTZMACHER, D. D. et al. Monitoramento de agrotóxicos em dois mananciais hídricos no Sul do Brasil. Revista Brasileira de Engenharia Agrícola e Ambiental, v. 12, n. 06, p. 632-637, 2008.

GUSTAFSON, D. I. Groundwater ubiquity score: a simple method for assessing pesticide leachability. Environmental Toxicology and Chemistry, v. 08, n. 04, p. 339-357,1989.

IGNJATOVIC, L. M. et al. Adsorptive stripping voltammetric determination of the herbicides atrazineand ametryne. Journal of the Serbian Chemical Society, v. 63, n. 01, p. 75-84, 1998.
KONSTANTINOU, I. K.; HELA, D. G.; ALBANIS, T. A. The status of pesticide pollution in surface waters (rivers and lakes) of Greece. Part I. Review on occurrence and levels. Environmental Pollution, v. 141, n. 03, p. 555-570, 2006.

MATALLO, M. B. et al. Lixiviação dos herbicidas tebuthiuron e diuron em colunas de solo. Pesticidas (UFPR), v. 13, p. 83-90, 2003.

MATALLO, M. B. et al. Sorption, degradation, and leaching of tebuthiuron and diuron in soil columns. Journal of Environmental Science Health, v. 40, n. 01, p. 39-43, 2005.

MILHOME, M. A. L. et al. Avaliação do potencial de contaminação de águas superficiais e subterrâneas por pesticidas aplicados na agricultura do Baixo Jaguaribe, CE. Engenharia Sanitária e Ambiental, v. 14, n. 03 p. 363-372, 2009.

THE PESTICIDE PROPERTIES DATABASE (PPDB). Developed by the Agriculture \& Environment Research Unit (AERU), University of Hertfordshire, funded by UK national sources and the EU-funded FOOTPRINT project (FP6 - SSP022704). 2010. Disponível em: <http://sitem.herts.ac.uk/aeru/ footprint/index2.htm>. Acesso em Acesso em: 15 abr. 2010.

SANCHES, S. M. et al. Pesticidas e seus respectivos riscos associados a contaminação da água. Pesticidas: Revista de Ecotoxicologia e Meio Ambiente, v. 13, p.53-58. 2003.

SERGIPE (Estado). Superintendência de Recursos Hídricos. Atlas Digital sobre os Recursos Hídricos de Sergipe. ARACAJU: SEPLANTEC-SRH, CD-ROM. 2004.

SILVA, A. de S. et al. Índice de sustentabilidade ambiental do uso da água (ISA_ÁGUA): municípios da região do entorno do rio Poxim, SE. Jaguariúna: Embrapa Meio Ambiente, 2004. 46 p.

SILVA, A. A. et al. Herbicidas: comportamento no solo. In: SILVA, A. A.; SILVA, J. F. (ed.). Tópicos em manejo de plantas daninhas. Viçosa: UFV, 2007. cap.5, p. 189-248.

SOARES, J. A. O Rio Poxim, processo urbano e meio ambiente. 2001. 67f. Monografia de Especialização (Gestão de Recursos Hídricos em Meio Ambiente). Universidade Federal de Sergipe, São Cristovão.

YOUNES, M.; GALAL-GORCHEV, H. Pesticides in Drinking Water - A Case Study. Food and Chemical Toxicology, v. 38, p. S87-S90, 2000. Suplemento.

WALLS, D.; SMITH, P. G.; MANSELL, M. G. Pesticides in groundwater in Britain. International Journal of Environmental Health Research, v. 06, n. 01, p. 55-62, 1996.

WATTS, D. W. et al. Storm flow export of metolaclor from a coastal plain watershed. Journal of Environmental Science Health, Part B, v. 35, p. 175-186, 2000. 